\title{
The electronic structure of manganese doped ceramic material $\mathrm{Ba}_{0.6} \mathrm{Sr}_{0.4} \mathrm{TiO}_{3}$
}

\author{
K. X. Hu ${ }^{*}$ and I. P. Jones ${ }^{*}$ \\ * Department of Metallurgy and Materials, University of Birmingham, Edgbaston, Birmingham, \\ B15 2TT, UK
}

\section{Introduction}

The electronic structure of $\mathrm{Mn}$ doped $\mathrm{Ba}_{0.6} \mathrm{Sr}_{0.4} \mathrm{TiO}_{3}$ ceramic materials has been determined by analyzing the low loss region of the electron energy loss spectrum in a transmission electron microscope. We calculate the interband transition strength which arises from maxima in the valence-band density of states via the dielectric function by a Kramers-Kronig transformation in the bulk and at segregated grain boundaries. The matrix and segregated boundaries are different in this regard.

\section{Experiment}

TEM specimens of $5 \mathrm{~mol} \% \mathrm{Mn}$ doped $\mathrm{Ba}_{0.6} \mathrm{Sr}_{0.4} \mathrm{TiO}_{3}$ were prepared by the conventional method of mechanical thinning followed by argon ion beam milling (GATAN PIPS). The measurements were performed with an Enfina PEELS system attached to a VG HB601UX FEG scanning transmission electron microscope (STEM) operating at $100 \mathrm{keV}$. The PEELS spectra were corrected for dark current and readout noise and the channel to channel gain variation. Under these conditions, the energy resolution was $0.45 \mathrm{eV}$ because of the cold field emission gun, the energy dispersion was $0.05 \mathrm{eV}$ and the probe size was $0.8 \mathrm{~nm}$ for the line scan measurement across the segregated boundaries. The grain boundary segregation was measured using STEM and EDX.

In order to obtain the single scattering distributions $S(E)$, the spectra acquired with the PEELS spectrometer were Fourier-Log deconvoluted to remove plural effects and then normalized using the method described by Egerton [1] to obtain the energy loss function $\operatorname{Im}(-1 / \varepsilon)$. The real and imaginary parts of the dielectric function were obtained, after removing surface loss effects, by an FFT-based Kramers-Kronig program. From the dielectric function the interband transition strength $\mathrm{J}_{\mathrm{cv}}$ was also calculated [2].

\section{Results and discussion}

Mn segregated boundaries were randomly selected, Fig. 1. The interband transition strength from matrix and boundaries is shown in Fig. 2. Because the dielectric constant changes at the grain boundary, the grain boundary might deserve to be regarded as a thin fillet of different material associated with a surface plasmon, although the material on either side has the same dielectric contant. The correct Kramers-Kronig Transformation is given by French [3] for boundary analyses. The interband transition strength presents a steep increase at an energy loss of about $2-3 \mathrm{eV}$ that may be attributed to the energy gap [4]. The interband transition strength from the segregated boundary is different from that of the bulk. There is no outstanding feature except for 2 peaks. Other notable features in the bulk (A, B and C), especially for the transitions with low energy near the band gap, become weaker at segregated grain boundaries. At A and B, there are 3 obvious peaks in the interband transition strength in the matrix. They are much weaker at the boundaries. Furthermore the two small peaks at B join into one broad peak. The characteristics of the matrix and boundaries are yet to be determined, but chemistry is an obvious candidate for the 
electronic structure changes. As shown in our previous results on spectral boundaries in pure $\mathrm{SrTiO}_{3}$, geometry is another possible cause to change the electronic structure [5]. However we found it happens only on some spectral boundaries. The chemical segregation is a more universal reason, since it is common to all segregated boundaries.

4. Conclusion

We have calculated the interband transition strength of $\mathrm{Ba}_{0.6} \mathrm{Sr}_{0.4} \mathrm{TiO}_{3}$ from the matrix and from segregated boundaries. It is proved that PEELS is a powerful analytical technique for the structure, bonding and electronic properties analysis of materials.

5. References

[1] R.F. Egerton, Electron Energy Loss Spectroscopy in the Electron Microscope, $2^{\text {nd }}$ Edition, Plenum Press, New York, 1996

[2] K. van Benthem, R.H. French, W. Sigle, C. Elsasser and M. Rühle. Ultramicroscopy 86 (2001) 303

[3] R.H. French, R.M. Cannon, L.K. DeNoyer and Y.-M. Chiang. Solid State Ionics 75 (1995) 13

[4] M.L. Bortz and R.H. French, Appllied. Spectroscopy. 43 (1989) 1498

[5] K.X.Hu and I.P.Jones Journal of physics 38 (2005) 183

6. Acknowledgements

We would like to thank Dr. Adam Papworth for help in operating the VG HB601UX FEG scanning transmission electron microscope (STEM) at the University of Liverpool (NWSTEM). We also would like to thank Dr. D. Zhang, Dr. B. Su and Dr. T. Button for help in preparing the $\mathrm{Ba}_{0.6} \mathrm{Sr}_{0.4} \mathrm{TiO}_{3}$ ceramic materials.

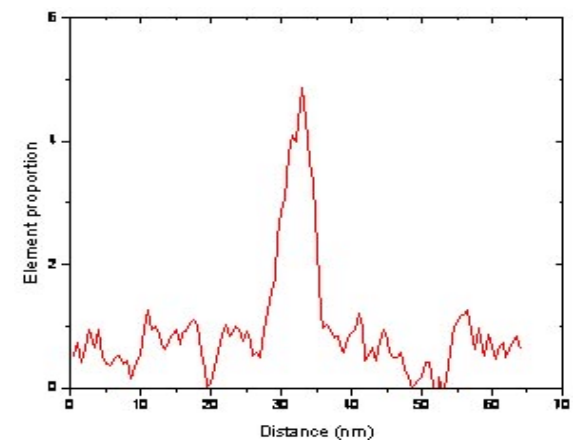

Fig.1. EDX linescan cross the manganese segregated grain boundary

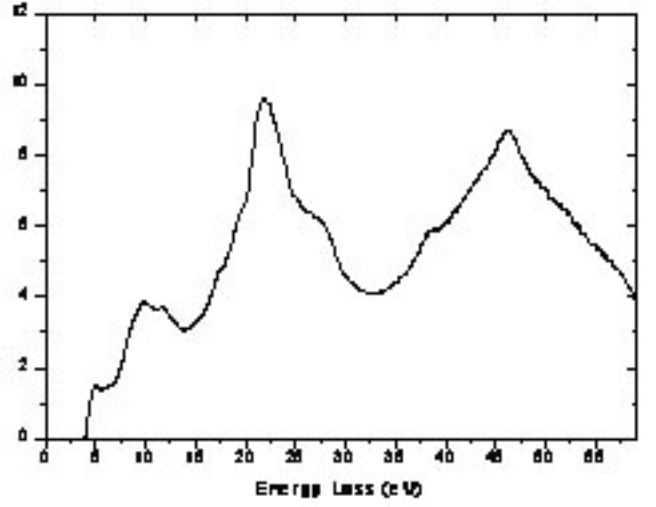

(a)

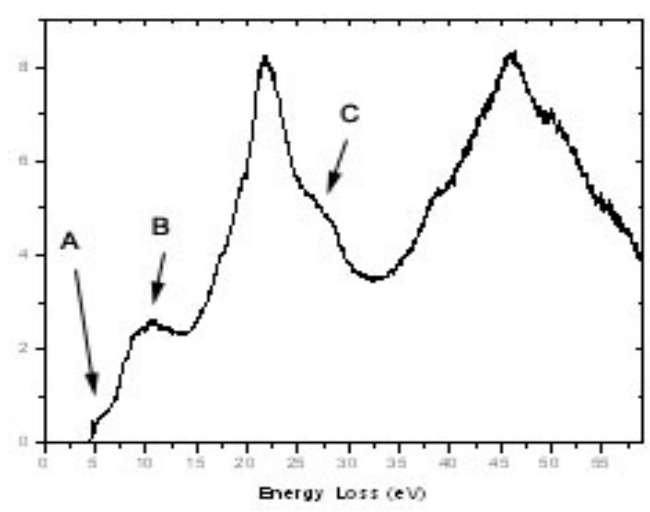

(b)

Fig.2. Interband band strength for $\mathrm{Ba}_{0.6} \mathrm{Sr}_{0.4} \mathrm{TiO}_{3}$ from matrix (a) and $\mathrm{Mn}$ segregated boundary (b) 